\title{
CLASSIFICAÇÃO ANDROLÓGICA POR PONTOS E CARACTERÍSTICAS ANDROLÓGICAS NA AVALIAÇÃO REPRODUTIVA DE TOUROS DA RAÇA GIR CANDIDATOS AO TESTE DE PROGÊNIE
}

\author{
ANDROLOGICAL POINTS CLASSIFICATION AND ANDROLOGICAL \\ REPRODUCTIVE EVALUATION CHARACTERISTICS IN GIR BULLS \\ CANDIDATES FOR PROGENIETEST
}

\author{
Juliano Bergamo Ronda ${ }^{1^{*}}$ ORCID - http://orcid.org/0000-0002-1882-5487 \\ Gustavo Lima Ribeiro ${ }^{1}$ ORCID - http://orcid.org/0000-0002-8779-176X \\ Jose Octavio Jacomini² ORCID - http://orcid.org/0000-0001-6930-3518 \\ Amanda Pifano Neto Quintal ${ }^{3}$ ORCID - http://orcid.org/0000-0001-6972-1568 \\ André Belico de Vasconcelos ${ }^{1}$ ORCID - http://orcid.org/0000-0003-1091-9531 \\ ${ }^{1}$ Universidade de Uberaba, Uberaba, MG, Brasil. \\ 2Universidade Federal de Uberlândia, Uberlândia, MG, Brasil. \\ ${ }^{3}$ Universidade Federal de Minas Gerais, Belo Horizonte, MG, Brasil. \\ "Autor para correspondência - juliano.ronda@uniube.br
}

\section{Resumo}

A eficiência reprodutiva deve ser avaliada em propriedades leiteiras para identificação de indivíduos com problemas reprodutivos ligados tanto à fêmea quanto ao macho, visando minimizar perdas na produção. A reprodução está entre os fatores que afetam a produtividade dos rebanhos e chama atenção, em alguns momentos, pelos baixos índices na pecuária tradicional. Objetivou-se estudar a ferramenta de avaliação espermática denominada índice CAP e sua correlação com as análises biométricas e morfológicas do sêmen de touros da raça Gir. Foram utilizados 34 touros da raça Gir (Bos taurus indicus) aptidão leiteira, hígidos, com 17 a 31 meses de idade e $450 \mathrm{~kg}$ de massa corporal média. Os touros foram submetidos a exame andrológico (avaliação física incluindo biometria testicular e morfológica do sêmen), seguido pela classificação andrológica por pontos (índice CAP). Foram realizadas três coletas independentes para avaliar a qualidade espermática por touro durante o período do experimento. De acordo com a avaliação do índice CAP, os animais foram classificados como excelentes $(17 \%)$ e muito bons $(83 \%)$. O índice CAP correlacionou-se positivamente com o perímetro escrotal e as características físicas do sêmen.

Palavras-chave: Andrologia, biometria, perímetro escrotal, sêmen, zebuínos

\begin{abstract}
Reproductive efficiency should be evaluated in dairy farms, when they are related to reproductive problems in both female and male. Breeding is among factors that affect herd productivity and draws attention by low rates of traditional livestock breeding. The aim was to study sperm evaluation tool known as the CAP index and its correlation with the biometric and morphological analyzes of Gir bulls semen. Thirty four healthy Gir bulls (Bos taurus indicus), dairy fitness, with 17 to 31 months of age with $450 \mathrm{~kg}$ of body mass were used. The bulls were submitted to andrological examination (physical evaluation including testicular biometry and semen morphological analysis) follow by andrological classification by points (CAP index). Three independent collections were carried out to evaluate sperm quality per bull during the experiment period According to the CAP index, animals were classified as excellent (17\%) and as very good (83\%). The CAP Index correlated positively with scrotal perimeter and semen physical characteristics.
\end{abstract}


Keywords: Andrology, biometrics, scrotal perimeter, semen, zebu.

Recebido em: 16 de dezembro de 2016.

Aceito em: 11 de dezembro de 2018

\section{Introdução}

A eficiência reprodutiva de machos e fêmeas deve ser avaliada em propriedades leiteiras para identificação de indivíduos com problemas reprodutivos visando minimizar perdas na produção. A reprodução está entre os fatores que afetam a produtividade dos rebanhos e chama atenção, em alguns momentos, pelos baixos índices reprodutivos na pecuária tradicional.

O número de animais subférteis e inférteis em serviço é de aproximadamente $40 \%$ (1). É chamado subfértil aquele animal, ou grupo de animais, no qual ocorre redução do potencial reprodutivo. Infértil é quando o touro apresenta, temporariamente, uma incapacidade reprodutiva. E estéril é o animal definitivamente incapacitado para a reprodução ${ }^{(2)}$.

O sêmen de alguns touros pode apresentar valores espermáticos satisfatórios, mesmo assim pode apresentar baixa taxa de fertilidade. Esse fato pode estar relacionado a aspectos morfométricos, fisiológicos (integridade da membrana plasmática) e bioquímicos (qualidade do DNA do espermatozoide) $)^{(3)}$.

Observa-se que o potencial reprodutivo de um touro é a soma de diversos fatores ligados à reprodução e, diante da importância das características andrológicas, notadamente aquelas ligadas ao perímetro escrotal (PE) e à qualidade do sêmen, têm sido propostos diferentes sistemas de pontuação que atuam como índices para a seleção andrológica.

Em vista disso, os touros podem ser avaliados e submetidos a sistemas de pontuação de tabelas por pontos que lhes confiram um ranking de classificação dependendo do seu potencial reprodutivo, como na classificação andrológica por pontos (CAP) $)^{(4)}$.

Nesse sentido, a utilização do índice CAP pode ter um papel importante no auxílio da seleção de touros melhoradores e podem-se associar, dentre os pontos já apresentados, fatores que podem ser inerentes à reprodução, como idade, puberdade, qualidade do sêmen, perímetro escrotal e componente genéticos.

O objetivo foi estudar o uso da ferramenta índice de CAP na avaliação reprodutiva de touros da raça Gir aptidão leiteira submetidos ao teste de progênie.

\section{Material e métodos}

Este experimento foi aprovado pelo Comitê de Ética em Experimentação Animal da Universidade de Uberaba (protocolo CEEA-076/2014).

Trinta e quatro touros da raça Gir (Bos taurus indicus) aptidão leiteira, hígidos, com 17 a 31 meses de idade e $450 \mathrm{~kg}$ de massa corporal média, foram submetidos a exame andrológico (avaliação física e morfológica do sêmen e biometria testicular) e a classificação andrológica por pontos. O potencial reprodutivo dos touros foi avaliado durante prova classificatória do teste de progênie ABCGIL/Embrapa, com duração de 150 dias, entre novembro de 2013 e abril de 2014.

Todos os animais receberam o mesmo manejo alimentar com $4 \%$ do peso corporal, em $\mathrm{kg}$ de (matéria seca) MS, disponível para todo o período experimental, sendo preconizado o consumo $2,5 \%$ do peso corporal, animal ${ }^{-1}$ dia $^{-1}$. O sistema de manejo dos touros no período foi semi-intensivo (lotação de 
1UA/ha), em regime de pastejo com lotação rotacionado, contendo cinco animais por piquete, com um total de sete piquetes, com mesma dimensão e predominância de capim do gênero Pannicum $s p$, e fornecimento de água e mistura mineral ad libitum, em área de lazer com sombreamento artificial de $3 \mathrm{~m}^{2} /$ animal.

Foram realizadas três coletas para o exame andrológico e qualidade espermática por touro durante o período experimental. As avaliações de biometria testicular foram realizadas com auxílio de fita métrica milimetrada (Walmur $80 \mathrm{~cm}$ ), com posicionamento na região mediana escrotal, no ponto de maior dimensão, envolvendo as duas gônadas e a pele escrotal.

Para a coleta do sêmen foi utilizado o método de eletroejaculação (EE), empregando-se o aparelho eletroejaculador modelo Walmur boijector 65A. Os ejaculados foram coletados em tubos coletores graduados, acoplados a funis previamente aquecidos em estufa mantida a $37^{\circ} \mathrm{C}$.

$\mathrm{Na}$ avaliação de volume, utilizou-se de proveta graduada e, para a obtenção da concentração espermática, foi utilizada a câmera de Neubauer. Para a contagem das células na câmara, o sêmen foi diluído na razão de 1:200, em solução de formol-salina-tamponada, e levado para a microscopia de campo claro com objetiva de 40X. Posteriormente, uma gota de sêmen a fresco foi colocada entre lâmina e lamínula previamente aquecidas a $37^{\circ} \mathrm{C}$ e em aumento de $400 \mathrm{x}$ para avaliação da motilidade espermática e do vigor segundo Diaset al. ${ }^{(2)}$. Para a avaliação morfológica das características dos espermatozoides, uma alíquota de sêmen de cada ejaculado foi acondicionada em tubos tipo ependorff contendo 1,0 mL de solução de formol-salina-tamponada ${ }^{(5)}$, em quantidade suficiente para turvar a amostra e esta foi estocada em temperatura ambiental e por período máximo de 48 horas. As análises morfológicas foram realizadas por meio de preparação úmida, com auxílio de microscopia de contraste de fase em aumento de 1000x, sob uma gota de óleo de imersão. Foram avaliadas 400 células por ejaculado, estas foram avaliadas quanto a defeitos espermáticos maiores, menores e totais, conforme os critérios utilizados por Blom ${ }^{(5)}$.

Após a avaliação andrológica, os touros foram classificados quanto aos seguintes critérios: perímetro escrotal - até 40 pontos; aspectos físicos (motilidade espermática progressiva retilínea e vigor espermático) - até 25 pontos; morfologia espermática (defeitos maiores e totais) - até 35 pontos, que, somados, permitiram obter a classificação final em questionáveis, bons, muito bons e excelentes reprodutores (Tabela 1).

Após a pontuação individual por característica, foi realizada a classificação andrológica por pontos, também denominada como índice CAP.

Assim, conforme descrito por Fonseca e colaboradores (1997), as características dos animais seguem de acordo com os aspectos físicos e morfológicos do sêmen e são relacionadas com o perímetro escrotal em função da faixa etária, o que estabelece a classificação em quatro categorias: excelentes; muito bons; bons, e questionáveis.

Para chegar ao valor final da pontuação no índice CAP no presente trabalho, utilizou-se a média das três coletas realizadas, para cada um dos parâmetros avaliados (Tabela 1).

$\mathrm{Na}$ análise estatística, foi utilizado o programa Graphpad Prism 6.0 (Graphpad software Inc., San Diego, USA). Os diferentes parâmetros avaliados como motilidade espermática progressiva retilínea, vigor espermático, volume, concentração espermática, morfologia espermática, perímetro escrotal e índice CAP foram expressos em estatística descritiva com média e desvio-padrão. Foi realizado o teste de correlação de Pearson, que permitiu correlacionar o índice CAP aos demais parâmetros analisados, ao nível de significância de 5\%. 
Tabela 1. Classificação andrológica por pontos sugerida para touros Bos taurus indicus, baseada nas características físicas, morfológicas do sêmen e no perímetro escrotal

\begin{tabular}{|c|c|c|c|c|}
\hline \multirow{2}{*}{ Parâmetros } & \multicolumn{4}{|c|}{ Classificação } \\
\hline & Excelente & Muito Bom & Bom & Questionável \\
\hline \multicolumn{5}{|l|}{ Motilidade Espermática } \\
\hline Vigor $(0-5)$ & 5 & $4<5$ & $3<4$ & $<3$ \\
\hline Motilidade (\%) & 75 & $60<75$ & $30<60$ & $<30$ \\
\hline Pontos Outorgados & 21a 25 & 16 a 21 & 10 a 16 & $<10$ \\
\hline \multicolumn{5}{|l|}{ Morfologia Espermática } \\
\hline Defeitos Maiores (\%) & 5 & $>5-10$ & $>10-20$ & $>20$ \\
\hline Defeitos Totais (\%) & 10 & $>10$ a 15 & $>15-30$ & $>30$ \\
\hline Pontos Outorgados & 30 a 35 & 25 a30 & 15 a25 & $>15$ \\
\hline \multicolumn{5}{|l|}{$\begin{array}{c}\text { Perímetro Escrotal }(\mathrm{cm}) \\
\text { Idade em meses }\end{array}$} \\
\hline $7-12$ & 21,0 & $19,5<21,0$ & $17,5<19,5$ & $<17,5$ \\
\hline $12-18$ & 26,0 & $24,0<26,0$ & $21,5<24,0$ & $<21,5$ \\
\hline $18-24$ & 31,5 & $28,5<31,5$ & $26,0<28,5$ & $<26,0$ \\
\hline $24-36$ & 35,0 & $32,0<35,0$ & $29,0<32,0$ & $<29,0$ \\
\hline $36-48$ & 37,0 & $33,5<37,0$ & $30,5<33,5$ & $<30,5$ \\
\hline$>48$ & 39,0 & $36,0<39,0$ & $33,0<36,0$ & $<33,0$ \\
\hline Pontos Outorgados & 35 a 40 & 25 a35 & 15 a 25 & $<15$ \\
\hline Índice CAP(Pontos) & $86 a 100$ & 66 a86 & 40 a66 & $<40$ \\
\hline
\end{tabular}

Fonte: Adaptado de Fonseca(6) - Índice CAP 86 a 100 pontos (excelentes); 66 a 86 pontos (muito bons); 40 a 66 pontos (bons); menor que 40 pontos (questionáveis).

\section{Resultados e discussão}

O valor médio de perímetro escrotal foi de $36,8 \pm 2,7 \mathrm{~cm}$, muito próximo dos valores verificados por Martinez et al. ${ }^{(7)}$, Pineda et al. ${ }^{(8)}$ e Gottschall, et al. ${ }^{(9)}$.

Hahn e colaboradores ${ }^{(10)}$ observaram correlação positiva entre a mensuração testicular e a concentração espermática em touros da raça Holandesa.

Palasz et al. (11), avaliando touros Bos taurus indicus, verificaram correlação positiva entre perímetro escrotal e produção diária de espermatozoides. No presente trabalho, não foi observada correlação entre concentração espermática e perímetro escrotal $(\mathrm{P}>0,05)$.

Quanto ao volume do ejaculado, o valor médio obtido no experimento foi de 4,2 $\mathrm{mL}$, estando semelhante ao trabalho reportado por Folhadella et al. ${ }^{(12)}$ e Silva et al. ${ }^{(13)}$. Todavia, é relatado na literatura que pequenas alterações no ejaculado podem ocorrer conforme o método de coleta ${ }^{(14)}$.

$\mathrm{Na}$ análise dos dados, não houve correlação entre concentração espermática e volume $(\mathrm{P}>0,05)$. Entretanto, a metodologia adotada para a coleta, apesar de poder alterar características macroscópicas, não diminui a representação das características microscópicas estudadas (motilidade, vigor, patologia 
espermática), tendo em vista que não há alteração nas espermatogêneses ${ }^{(15)}$.

Valentim et al ${ }^{(16)}$ descreveram que a fisiologia funcional depende principalmente do diâmetro e do número de túbulos seminíferos e que a variação da característica pode estar associada à idade com que os touros atingem a maturidade sexual e não à forma de coleta.

Vince e colaboradores ${ }^{(17)}$ vão mais além e apontam que fatores intrínsecos como o ambiente, a raça e a idade podem alterar de forma significativa parâmetros andrológicos, como motilidade e concentração espermática. $\mathrm{O}$ que corrobora os relatos de Thundathil et al ${ }^{(18)}$, em que a taxa de fertilidade pode variar em até $20 \%$ entre touros classificados como excelentes reprodutores, pois há alterações nos aspectos microscópicos do sêmen.

O valor médio obtido para a concentração espermática por $\mathrm{mL}$ de sêmen de espermatozoides no ejaculado foi de $967,6 \times 10^{6}$ e volume total de 4,2mL (Tabela 2). Os valores de concentração espermática foram superiores aos valores reportados por Santos et al. ${ }^{(6)}$ Já o número total de espermatozoides no ejaculado apresentaram-se dentro dos valores estabelecidos por Martinez et $\mathrm{al}^{(7)} \mathrm{e}$ Salvador et al. ${ }^{(19)}$. Corroborando-se, assim, os valores médios estabelecidos pelo Colégio Brasileiro de Reprodução Animal ${ }^{(20)}$ mínimo365x 10\% $/ \mathrm{ml}$, para a concentração, e volume total do ejaculado (5-8mL).

Tabela 2. Média e desvio-padrão $(\mathrm{X} \pm \mathrm{SD})$ das avaliações andrológicas de três ejaculados coletados por eletroejaculação (EE) de 34 touros da raça Gir aptidão leiteira

\begin{tabular}{lccc}
\hline Avaliações andrológicas & $\mathbf{X} \pm \mathbf{D P}$ & Min. & Max \\
\hline Perímetro Escrotal $(\mathrm{cm})$ & $36,4 \pm 2,7$ & 32 & 43 \\
Volume (mL) & $4,2 \pm 1$ & 3,0 & 7,3 \\
Concentração espermática $\left(\mathrm{x} 10^{6 / \mathrm{mL})}\right.$ & $967,6 \pm 367,7$ & 317,5 & 1725,0 \\
Motilidade (\%) & $75,5 \pm 12,3$ & 50 & 90 \\
Vigor (0-5) & $3,5 \pm 0,8$ & 3,0 & 5,0 \\
Defeitos totais (\%) & $6,5 \pm 1,4$ & 3,0 & 10,0 \\
Idade (meses) & $25,8 \pm 4,5$ & 17 & 31 \\
\hline
\end{tabular}

Legenda: $\mathrm{X}=$ média; $\mathrm{DP}=$ Desvio-padrão; Mín. = mínima; Máx $=$ Máxima.

O valor médio para motilidade e vigor foi de 75,5\% e 3,5 (Tabela 2), respectivamente, que corresponde, na classificação andrológica (Tabela 1), a muito bom e bom, respectivamente, de acordo com Fonseca ${ }^{(6)}$.

Após a avaliação da correlação entre motilidade e vigor, verificou-se que esta foi positiva ( $\mathrm{r}=0,72-$ $\mathrm{P}<0,05)$, semelhante à verificada por Vale Filho ${ }^{(4)}$ e Martinez et al. ${ }^{(7)}$.

A correlação entre motilidade e vigor pode ser explicada por serem variáveis dependentes do metabolismo espermático ${ }^{(5)}$. Por conseguinte, também foi observada correlação significativa entre motilidade e defeitos totais $(\mathrm{r}=0,40 \mathrm{P}<0,05)$, como descrito na Tabela 3.

A correlação baixa encontrada entre motilidade e defeitos totais define-se por serem variáveis dependentes. A literatura registra que uma alteração na espermatogênese, como um aumento da temperatura ambiente ou corpórea, promove de forma significativa uma alteração na qualidade morfológica das células espermáticas, com interferência na produção e na viabilidade do espermatozoide ${ }^{(17)}$.

De acordo com a avaliação do índice CAP, foi observado que $17 \%$ dos animais apresentaram classificação como excelentes e $83 \%$ foram classificados como muito bons. A classificação andrológica por pontos correlacionou-se positivamente $(\mathrm{P}<0,05)$ com o perímetro escrotal e as características físicas do sêmen.

Verificaram-se as correlações entre CAP e perímetro escrotal $(\mathrm{r}=0,53)$; entre CAP e motilidade $(\mathrm{r}=0,42)$; entre CAP e vigor espermático $(\mathrm{r}=0,55)$, e entre CAP e defeitos totais $(\mathrm{r}=0,14)$ (Tabela 3$)$. $\mathrm{Na}$ correlação de CAP e idade, observou-se que não houve correlação ( $p>0,05)$. 
Tabela 3. Correlação da CAP com parâmetros de motilidade espermática progressiva retilínea, vigor espermático e defeitos totais

\begin{tabular}{ccccccc}
\hline Avaliações/Avaliações & CAP & PE & MOT & DEF & VIGOR & IDADE \\
\hline CAP & - & $0,53^{*}$ & $0,42^{*}$ & $0,14^{*}$ & $0,55^{*}$ & 0,01 \\
PE & $0,53^{*}$ & - & 0,06 & 0,004 & 0,11 & $-0,07$ \\
MOT & $0,42^{*}$ & 0,06 & - & $0,40^{*}$ & $0,72^{*}$ & $-0,08$ \\
DEF & $0,14^{*}$ & 0,004 & $0,40^{*}$ & - & $0,37^{*}$ & 0,09 \\
VIGOR & $0,55^{*}$ & 0,11 & $0,72^{*}$ & $0,37^{*}$ & - & $-0,03$ \\
IDADE & 0,01 & $-0,07$ & $-0,08$ & 0,09 & $-0,03$ & - \\
\hline
\end{tabular}

Teste de correlação de Pearson, $\mathrm{O}$ símbolo *define P $<0,05$, CAP - Classificação andrológica pontos; PE Perímetro escrotal; MOT - Motilidade espermática progressiva retilinea; DEF, TOT - Defeitos totais; VIGOR - Vigor espermático.

A correlação entre perímetro escrotal e CAP $(\mathrm{r}=0,53-\mathrm{P}<0,05)$ corrobora de forma positiva as avaliações de defeitos totais e CAP $(\mathrm{r}=0,14-\mathrm{P}<0,05)$, mesmo que o coeficiente de correlação seja menor, quando comparado ao perímetro escrotal. Esta análise é justificada, uma vez que não há correlação entre o perímetro escrotal e defeitos totais $(\mathrm{P}>0,05)$. Este resultado também foi reportado por Johnson et al, ${ }^{(21)}$ que encontraram correlação semelhante do CAP com defeitos totais.

Assim como Vale Filho(4), este trabalho enfatiza que a relação do CAP, como um sistema de avaliação, por sua facilidade e praticidade de programação, pode ser utilizado na seleção de bovinos da raça Gir com aptidão leiteira.

\section{Conclusões}

Com base nos resultados encontrados, concluiu-se que a classificação andrológica por pontos apresenta correlação positiva com perímetro escrotal e características físicas do sêmen, sendo possível e importante o seu uso como ferramenta na avaliação reprodutiva de touros da raça Gir aptidão leiteira, candidatos ao teste de progênie.

\section{Agradecimentos}

Agradecemos à Associação Brasileira de Criadores de Gir (ABCGIL), pela parceria e pelo apoio na execução do presente trabalho, e à Fundação de Amparo à Pesquisa do Estado de Minais Gerais (FAPEMIG).

\section{Referências}

1. Lopes FG, Junior CK, Ferreira M,B, Menegassi SRO, Guidoni PB, Correa LS, Avaliação da maturidade sexual e classificação andrológica por pontos (CAP) de touros Jovens da raça Nelore, criados no noroeste do estado do Paraná, Uniciência [Periódico na internet], 2016; 20:108-112, Disponível em: http://www.pgsskroton.com.br/seer/index.php/uniciencias/article/view/4393/3375

2. Dias JC, Andrade VJ, Martins JAM, Emerick LL, Gonçalves PEM, Filho VRV, Classificação andrológica por pontos (CAP) de touros Nelore (Bos taurus indicus) de dois e três anos de idade, criados sob pastejo, Ciência Animal Brasileira [Periódico na internet], 2009;10:1094-1099, Disponível em: https://www.revistas.ufg.br/vet/article/view/3950/5923

3. Boe-Hansen GB, Fortes MRS, Satake N, Morphological defects, sperm DNA integrity, and protamination of bovine spermatozoa, Andrology [Periódico na internet], 2018; 1-7, doi: 10.1111/andr.12486 
4. Vale Filho, V.R. Subfertilidade em touros: parâmetros para avaliação andrológica e conceituação geral. Cadernos Técnicos de Veterinária e Zootecnia [Periódico na internet] ,2001;35:81-87, Disponível em: http://www.scielo.br/scielo.php?script=sci_nlinks\&ref=000110\&pid=S0102-0935201200030000300022

5. Blom E, Ultrastructure of some characteristic sperm defects and a proposal for a new classification of the bull spermiogram, Nordisk veterinary medicin, 1973;25:383-39.

6. Fonseca, VO, Santos, NR, Malinski, PR. Classificação andrológica de touros zebus (Bos taurus indicus) com base no perímetro escrotal e características morfo-físicas do sêmen. Revista Brasileira de Reprodução Animal, v.21, n.2, p.36-39, 1997.

7. Martinez ML, Verneque RS, Teodoro RL, Oliveira Paula LR, Cruz M, Campos JP, Rodrigues LH, Oliveira J, Vieira F, Bruschi JH, Durães MC, Correlações entre Características da Qualidade do Sêmen e a Circunferência Escrotal de Reprodutores da Raça Gir, Rev. bras. Zootec[Periódico na internet], 2000: 29(3):700-706, Disponível em: http://www.scielo.br/pdf/\%0D/rbz/v29n3/5812.pdf

8. Pineda NR, Fonseca VO, Proença RV, Potencial reprodutivo de touros Nelore: libido, capacidade de serviço e eficiência em acasalamento com elevada proporção de vacas, Revista Brasileira de Reprodução Animal, 2000;24:44-51, Disponível em: http://cbra.org.br

9. Gottschall CS, Mattos RC, Achados de exames andrológicos em touros de corte Bos taurus e Bos indicus, Revista Brasileira de Reprodução Animal, 1997;21:25, Disponível em: http://cbra.org.br

10. Hahn J, Foote RH, Seidel GE, Testicular growth and related sperm outputin dairy bulls, Journal of Animal Science[Periódico na internet],1969;29:41-47, Disponivel em: https://www.ncbi.nlm.nih.gov/pubmed/5356694

11. Palasz AT, Cates WF, Barth AD, Mapletoft RJ, The relationship between scrotal circumference and quantitative testicular traits in yearling beef bulls, Theriogenology [Periódico na internet], 1994; 42: 715-726, Disponível em: https://www.ncbi.nlm.nih.gov/pubmed/16727577

12. Folhadella IM, Sá WF, Ferreira AM, Camargo LSA, Viana JHM, Ramos AA, Silva MVGB, Características andrológicas de touros da raça Gir, Arquivo Brasileiro Medicina Veterinária e Zootecnia [Periódico na internet], 2006;58:809-815, Disponível em: http://www.scielo.br/pdf/abmvz/v58n5/16.pdf.

13. Silva AR, Ferraudo AS, Perecin D, Lima VFMH, Efeito da idade do touro e do período de colheita de sêmen sobre as características físicas e morfológicas do sêmen de bovinos de raças européias e zebuínas, Revista Brasileira de Zootecnia [Periódico na internet], 2009;38:1218-1222, Disponível em: http://www.scielo.br/pdf/rbz/v38n7/v38n7a09.pdf

14. Persson Y, McGowan M, So“ derquist L Comparison between the Sperm Morphology in Semen Samples Obtained from Yearling Beef Bulls by Transrectal Massage of the Ampullae and Cauda Epididymal Dissection, Reprod Dom Anim [Periódico na internet], 2006; 41, 233-237, Disponivel em: doi 10.1111/j.1439$\underline{0531.2006 .00675 . x}$

15. Bhattacharyya HK, Goswami BK, K,M, Bujarbaruah KM, Deka BC,Biswas RK, Collection and characterization of semen in Mithun (Bos frontalis) bulls, Theriogenology [Periódico na internet],2009; 72:699-703, Disponível em: 10.1016/j.theriogenology.2009.05.010

16. Valentim R, Aruda RP, Barnabe RC, Biometria testicular de touros nelore (Bos taurus indicus) e touros cruzados Nelore europeu (Bos taurus indicus X Bos taurus taurus) aos 20 e 24 meses de idade, Braz, J, Vet, Res, Anim, Sci [Periódico na internet], 2002;39:113-120, Disponível em: http://www.scielo.br/pdf/bjvras/v39n3/15811.pdf

17. Vince s, Zura-Zaja I, Samardzija M, Majic Balic I, Vilic M, Duricic D, Milinkovic-Tur S, Age-related differences of semen quality, seminal plasma, and spermatozoa antioxidative and oxidative stress variables in bulls during cold and warm periods of the year, Animal, 2018, 12(3):559-568, Disponível em: doi: $\underline{10.1017 / \text { S1751731117001811 }}$

18. Thundathil JC, Dance AL, Kastelic JP, Fertility management of bulls to improve beef cattle productivity. Theriogenology. 2016;86:397-405.

19. Salvador DF, Andrade VJ, Vale Filho VR, Dias JC, Nogueira LAG, Associação entre o perfil andrológico 
e a congelação de sêmen de touros da raça Nelore aos dois anos de idade, pré-selecionados pela classificação andrológica por pontos (CAP), Arquivo Brasileiro de Medicina Veterinária e Zootecnia [Periódico na internet], 2008;60:587-593, Disponível em: http://www.scielo.br/pdf/abmvz/v60n3/10.pdf

20. Manual para exame andrológico e avaliação de sêmen animal/Colégio Brasileiro de Reprodução Animal, 3,ed, - Belo Horizonte: CBRA 2013.Disponível em: www.cbra.org.br

21. Johnson WH, Thompson JA, Kumidiaka J, The determination and correlation of reproductive parameters of performance-tested Hereford and Simmental bulls, Theriogenology [Periódico na internet], 1995:44:973982, Disponível em: https://www.ncbi.nlm.nih.gov/pubmed/16727792 